\title{
Eight-Channel Optical Add Drop Multiplexer Based on Ring Resonator Using LNOI Channel Waveguides
}

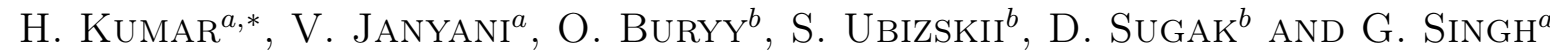 \\ ${ }^{a}$ Department of Electronics and Communication Eng., MNIT, Jaipur, India \\ ${ }^{b}$ Lviv Polytechnic National University, Lviv, Ukraine
}

\begin{abstract}
In this paper we report on an eight-channel optical add drop multiplexer based on ring resonator using lithium niobate on insulator channel waveguides. It is suitable for a DWDM-GPON network with channel spacing of $100 \mathrm{GHz}$ in C-band and data rate is 10 Gbps. The insertion loss at the drop port is maximum $1.2 \mathrm{~dB}$ and the $Q$-factor is 1636 . It can be used as multiplexer as well as demultiplexer in 8-channel DWDM systems.
\end{abstract}

DOI: 10.12693/APhysPolA.133.997

PACS/topics: 42.79.Sz

\section{Introduction}

The high speed all optical networks required costeffective and energy-efficient technology for optical signal processing devices like optical add drop multiplexer (OADM), optical filters, all optical switches etc. Silicon photonics is well developed and many devices is fabricated using silicon on insulator (SOI) [1]. The ring resonators are cascaded to make all optical multiplexer and demultiplexer for DWDM systems. The higher order microring resonators are used to reduce the cross-talk in OADM [2]. Lithium niobate on insulator (LNOI) is the emerging technology for photonics devices as it shows an extensive electro-optic (EO), acousto-optic ( $\mathrm{AO}$ ) and thermo-optic (TO) effects, which help in making tunable devices $[3,4]$. The lithium niobate (LN) based ring resonator can be easily made tunable by using electricoptic properties of LN [5]. Microring resonators are also used to make all optical logic devices like logic gates, encoder [6]. In this paper, eight microring resonators based on LNOI are cascaded to form the PIC of eight channel OADM. Single channel OADM is designed and simulated using finite difference time-domain (FDTD) method.

\section{Optical add drop multiplexer}

The PIC of 8-channel OADM is made of eight cascaded ring resonators. The block diagram of 8 channel OADM using microring resonator is shown in Fig. 1; each consists of two bus waveguide which are coupled by a ring waveguide. It is a four port device, for demultiplexer we are using input port, through port and drop port and for multiplexer input port, through port and add port is used. The dropped optical power, $P_{\text {drop }}$ is calculated in terms of input power, $P_{i n}$ and coupling coefficients, $t_{11}$ and $t_{12}$ by using Eq. (1):

\footnotetext{
*corresponding author; e-mail: 2015pwc5305@mnit.ac.in
}

$$
P_{\text {drop }}=P_{\text {in }} \frac{\left|t_{12}\right|^{4}}{\left|1-t_{11}^{2} \mathrm{e}^{\mathrm{j} \beta L}\right|^{2}},
$$

where $L$ is the effective length of ring waveguide and $\beta$ is the phase constant along the ring waveguide. At the resonance the phase difference after a round trip is integer multiple of $2 \pi$. The main stream data in form of optical signal is flowing from input port to through port and when it is used as demultiplexer, the individual channel data is received at drop port. In multiplexer configuration, add port is used to multiplex the channels to form the DWDM main stream optical signal. The refractive contrast is high enough so that bending loss and propagation loss are negligible.

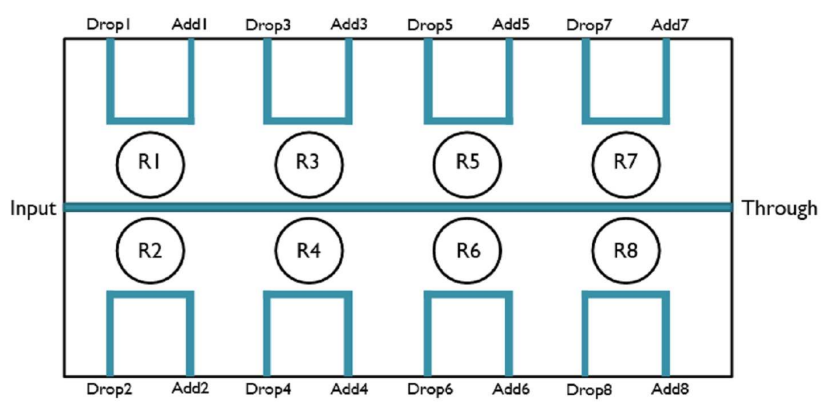

Fig. 1. The block diagram of 8-channel OADM.

\section{Design implementation of 8-channel OADM}

The PIC of eight channel OADM based on optical ring resonator is designed for the DWDM system with a channel spacing $100 \mathrm{GHz}(0.8 \mathrm{~nm}$ at $1550 \mathrm{~nm})$ and we wanted to drop every 8th channel i.e. the required free spectral range (FSR) is $800 \mathrm{GHz}(6.4 \mathrm{~nm})$. The layout of design is shown in Fig. 2. The radius of ring is $59.7 \mu \mathrm{m}$ hence effective length $L=375 \mu \mathrm{m}$.

The design parameters are described in Table I. Each microring is designed using lithium niobate as substrate then a thin layer of $\mathrm{SiO}_{2}$ on which OADM is designed using lithium niobate channel waveguide. The waveguide 


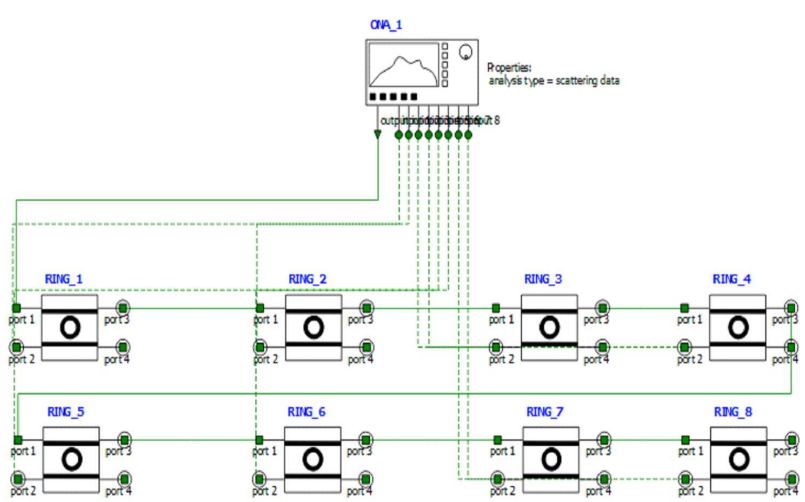

Fig. 2. Design layout of PIC of 8-channel OADM.

width is $700 \mathrm{~nm}$ and height is $350 \mathrm{~nm}$. The separation between the bus waveguide and ring waveguide is $100 \mathrm{~nm}$. The refractive indexes of $\mathrm{LN}$ and $\mathrm{SiO}_{2}$ are taken at $1.55 \mu \mathrm{m}$. The fundamental mode is confined in the waveguide as shown in Fig. 3. The mode is confined which means that the optical signal is propagating through the waveguide core and leakage signal is less. The TE polarization is used in simulation.

TABLE I

Design parameters of ring resonator.

\begin{tabular}{c|c|c}
\hline \hline Name & Value & Description \\
\hline W_core & $700 \mathrm{~nm}$ & waveguide width \\
\hline $\mathrm{H}$ _core & $350 \mathrm{~nm}$ & waveguide height \\
\hline R0 & $59.7 \mu \mathrm{m}$ & radius of curvature \\
\hline $\mathrm{N}$ _core & 2.211 & $\mathrm{LN}$ refractive index \\
\hline $\mathrm{N}$ _clad & 1.444 & $\mathrm{SiO}_{2}$ refractive index \\
\hline Dx & $100 \mathrm{~nm}$ & separation b/w waveguides
\end{tabular}

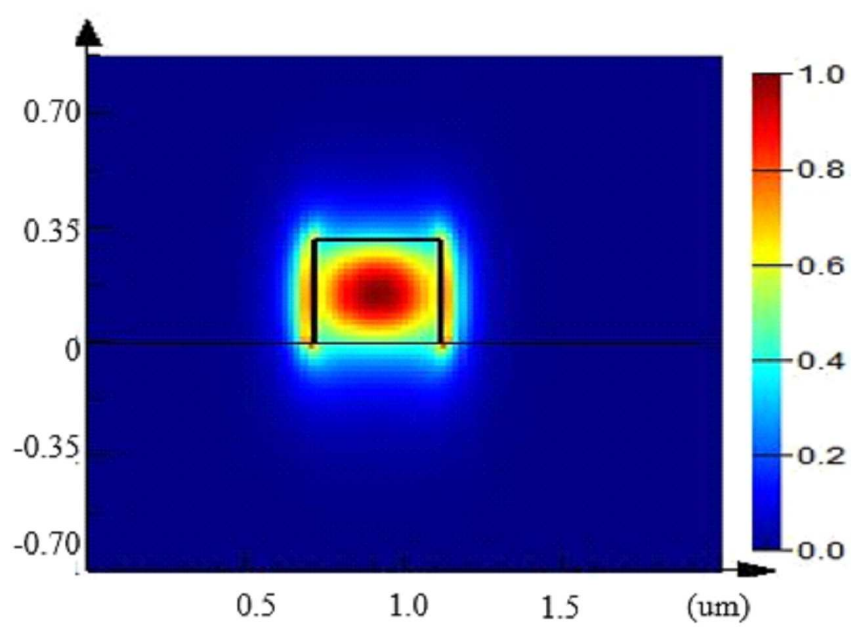

Fig. 3. Fundamental mode of propagation in channel waveguide.

\section{Results}

The OADM designed using LNOI and simulated using FDTD method. The PIC is simulated on the Lumerical Interconnect tool using the scattering data analysis. The transmission curve is observed at drop port of each ring and plotted in fig. 4. It is observed the insertion loss at the drop port is maximum $1.2 \mathrm{~dB}$ when used as demultiplexer and the Q-factor is 1636 . The data rate is 10 Gbps. The crosstalk is also minimal and at the resonate condition optical power at other ports is zero.

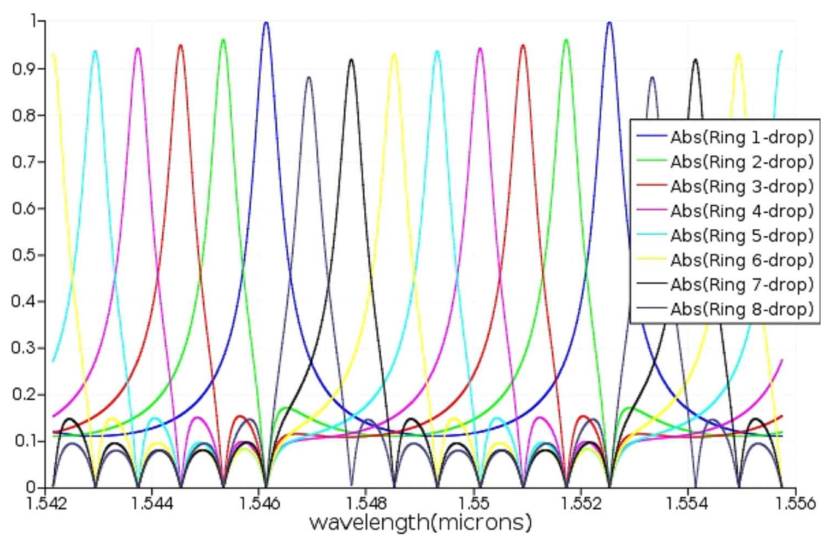

Fig. 4. Transmission plot at drop port of each ring.

The transmission plot at the through port of 8-channel OADM as DEMUX when four channels are dropped and at the through port of 8-channel OADM as MUX when those 4 channels are added again is shown in Fig. 5 .

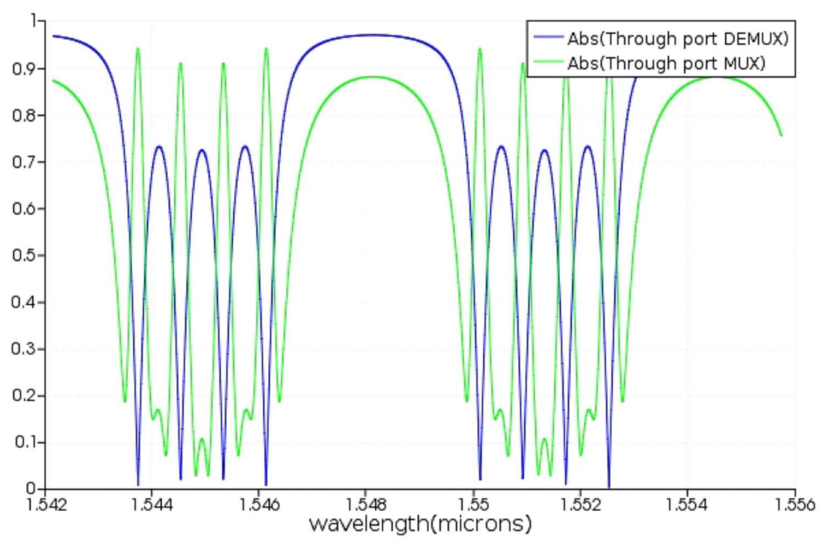

Fig. 5. Transmission plot at through port of DEMUX and MUX.

\section{Conclusions}

In this work the structuring of all optical add drop multiplexer based on microring resonator is done by using the LNOI and cascaded to form PIC of 8-channel OADM. The OADM is designed for a $100 \mathrm{GHz}$ channel spacing DWDM system. The insertion loss at the drop port is 
maximum $1.2 \mathrm{~dB}$ and the $Q$-factor is 1636 . It can be used as multiplexer as well as demultiplexer in 8-channel DWDM systems. The data rate is $10 \mathrm{Gbps}$ and hence this PIC can be used in DWDM-GPON network to enhance the performance.

\section{Acknowledgments}

The authors are grateful to India-Ukraine intergovernmental science \& technology cooperation programme between the MNIT Jaipur (India) and the Lviv Polytechnic National University, Lviv (Ukraine) for technical support. Project sanction no.: INT/RUS/UKR/P$15 / 2015$. The work was partially supported by the Ukrainian Ministry of Education and Science (project DB/MEZHA no. 0118U000273).

\section{References}

[1] D. Wu, Y. Wu, Y. Wang, J. An, X. Hu, Opt. Commun. 354, 386 (2015).

[2] S. Papaioannou, G. Dabos, K. Vyrsokinos, G. Giannoulis, A. Prinzen, C. Porschatis, M. Waldow, D. Apostolopoulos, H. Avramopoulos, N. Pleros, Opt. Commun. (ECOC), 1 (2014).

[3] H. Kumar, V. Janyani, B. Oleh, U. Serhij, S. Dmytro, G. Singh, Int. Conf. on Fibre Optics and Photonics, W3A-15 (2016).

[4] G. Poberaj, H. Hu, W. Sohler, P. Günter, Laser Photon. Rev. 64, 488 (2012).

[5] Tzyy-Jiann Wang, Chia-Hong Chu, Che-Yung Lin, Opt. Lett. 32, 2777 (2007).

[6] A. Godbole, P.P. Dali, V. Janyani, T. Tanabe, G. Singh, IEEE J. Sel. Top. Quant. Electron. 22, 326 (2016). 